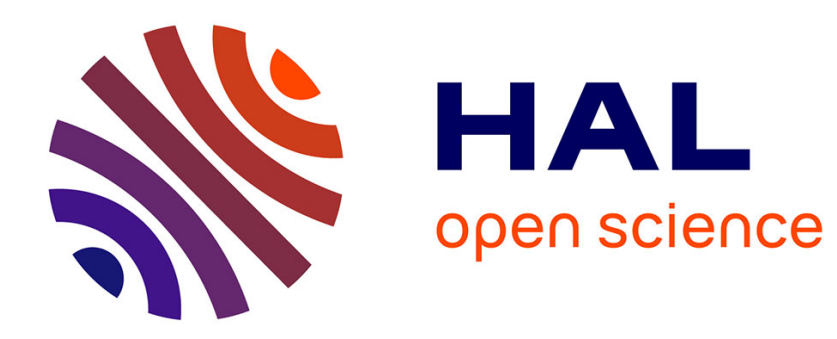

\title{
Traumatisme de l'inceste et destin du féminin
}

\author{
A.-V. Mazoyer, J.-P. Martineau
}

\section{To cite this version:}

A.-V. Mazoyer, J.-P. Martineau. Traumatisme de l'inceste et destin du féminin. Annales MédicoPsychologiques, Revue Psychiatrique, 2011, 169 (6), pp.383. 10.1016/j.amp.2011.05.004 . hal00771586

\section{HAL Id: hal-00771586 https://hal.science/hal-00771586}

Submitted on 9 Jan 2013

HAL is a multi-disciplinary open access archive for the deposit and dissemination of scientific research documents, whether they are published or not. The documents may come from teaching and research institutions in France or abroad, or from public or private research centers.
L'archive ouverte pluridisciplinaire HAL, est destinée au dépôt et à la diffusion de documents scientifiques de niveau recherche, publiés ou non, émanant des établissements d'enseignement et de recherche français ou étrangers, des laboratoires publics ou privés. 


\section{Accepted Manuscript}

Title: Traumatisme de l'inceste et destin du féminin

Authors: A.-V. Mazoyer, J.-P. Martineau

PII: $\quad$ S0003-4487(11)00130-2

DOI: $\quad$ doi:10.1016/j.amp.2011.05.004

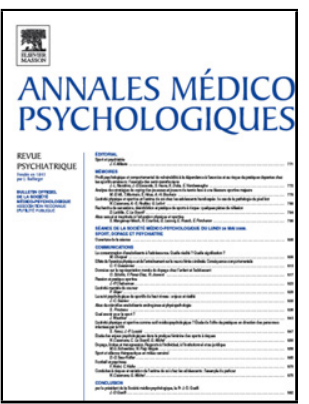

Reference: $\quad$ AMEPSY 1333

To appear in: $\quad$ Annales Médico-Psychologiques

Please cite this article as: Mazoyer A-V, Martineau J-P, Traumatisme de l'inceste et destin du féminin, Annales medio-psychologiques (2010), doi:10.1016/j.amp.2011.05.004

This is a PDF file of an unedited manuscript that has been accepted for publication. As a service to our customers we are providing this early version of the manuscript. The manuscript will undergo copyediting, typesetting, and review of the resulting proof before it is published in its final form. Please note that during the production process errors may be discovered which could affect the content, and all legal disclaimers that apply to the journal pertain. 


\title{
Communication
}

\section{Traumatisme de l'inceste et destin du féminin}

Fate of feminine to female adolescent incest victim

\author{
A.-V. Mazoyer ${ }^{\text {a, }}$, J.-P. Martineau ${ }^{\text {b }}$ \\ ${ }^{a}$ Psychologue, Maître de conférences, UFR de psychologie, Université Toulouse II, \\ Laboratoire LCPI, Toulouse, France \\ ${ }^{b}$ Professeur émérite des Universités, Université Montpellier III, France
}

Auteur correspondant: A.-V. Mazoyer, Psychologue, Maître de conférences, UFR de psychologie, Université Toulouse II, Laboratoire LCPI, 5, allées Antonio-Machado, 31058 Toulouse cedex 9, France

Adresse mail : avaleriemazoyer@orange.fr

\section{Résumé}

À la surrection pubertaire peut s'ajouter la violence du traumatisme sexuel, entravant ce possible moment de reconstruction narcissique chez la fille qu'est l'adolescence. Nous nous proposons d'analyser le possible investissement du féminin chez une jeune fille prépubère abusée sexuellement, enfant, par son père. Considérant le refoulement des représentations liées à l'inceste, nous posons que la répression affecte l'investissement possible du corps féminin et son activité de contenance et de réceptivité.

Nous nous sommes référés à trois études utilisant la méthodologie projective, portant sur des adolescentes (consultantes et tout-venant) et des femmes anciennement maltraitées physiquement ou abusées sexuellement.

Chez notre sujet, les épreuves projectives (Rorschach et Thematic Aperception Test) attestent la répression du féminin, un manque de dynamisme et de mobilité de la pensée, et le refus d'engagement dans la relation avec l'autre. La question de l'identité sexuelle semble éludée par la problématique de l'identité, la cruauté envers soi et les autres étant une manière de se protéger de tout lien.

Mots clés : Adolescence; Épreuves projectives ; Féminin ; Féminité ; Inceste 


\begin{abstract}
In the juvenile surrection can be added the violence of the sexual traumatism, hindering this possible moment of narcissistic reconstruction to an incest victim.

We suggest analyzing the possible investment of female identity (envelope feminity and orifice feminity) to a pre-pubescent girl, victim of childhood incest.

We put the hypothesis which the expulsion of the representations bound to the incest affects the possible investment of the feminine body and its containment function.

We referred to three studies, using projective tests, concerning teenagers (some were hospitalized after a suicide attempt and te others had no psychiatric care) and women, victims of childhood incest or which suffered from maltraitant in childhood. To our subject, the projective tests (Rorschach and Thematic Aperception Test) give evidence of the repression of the female identity, a lack of dynamism and mobility of the thought and relational problems.

The question of the sexual identity seems evaded by the problem of the identity, the cruelty to one and others being a way of protecting herself from any link.
\end{abstract}

Keywords: Adolescence; Feminine; Feminity; Incest; Projective test

\title{
1. Introduction
}

Qu'en est-il de l'élaboration du féminin ${ }^{1}$ chez une adolescente placée et ayant été abusée, lorsque la problématique de l'adolescence est aggravée par la réactivation de traumas antérieurs ? Selon Bigras et Balasc [3, p. 56] : «[...] Se profile la question posée à la victime de l'inceste, de sa place à retrouver, de son identité à reconstruire, après ce passage où elle a pu se vivre comme n'étant plus elle-même, mais une autre. »

La problématique ici mise en exergue concerne la mise en travail des polarités du féminin et de la féminité à l'adolescence. Avec l'étayage d'épreuves projectives (Rorschach, TAT), nous nous proposons d'apprécier l'investissement du féminin à l'aube de l'adolescence chez une jeune fille abusée pendant son enfance par son père. Bernardi et Peguet [2, p. 244] analysent l'impact de l'inceste sur le travail mental en termes de «suspension de l'activité idéique et de l'activité fantasmatique». Dans cette logique et au vu de la présentation enfantine de notre sujet (bientôt 13 ans), nous posons que la répression des représentations liées à l'inceste affecterait aussi l'activité psychique requise pour l'appropriation du corps 
féminin adolescent. Cela s'oppose à une hypothèse qui rechercherait dans les protocoles projectifs des réponses complexuelles à caractère sexuel au regard de la corrélation établie entre conduites sexuelles à risques et antécédents d'abus sexuels [14].

\section{Création du féminin à l'adolescence}

La construction de l'identité de la femme est double : éclosion de la féminité et inclusion du féminin. Il est d'abord, au niveau le plus manifeste, investissement de l'enveloppe, où l'épanouissement des courbes et des volumes est en attente d'un regard qui confirme cette féminité. Elle offre ses formes rayonnantes, sa convexité provocante, au plus près de la distinctivité phallique, érectile [5]. Parures et parades de la féminité éclatante conservent au secret le féminin obscur. Ainsi, l'épreuve de l'adolescence consiste-t-elle à intégrer l'expérience de passivité féminine [12], ou encore d'un investissement positif d'un réceptacle en attente de révélations de sa capacité d'ancrage et de création; non sans crainte pour la fille qui a subi une emprise incestueuse. En effet, pour elle, ce réel traumatique contamine d'effroi le travail du féminin formulé ainsi: "Comment intégrer ces transformations corporelles qui s'approchent dangereusement de la scène primitive et de la réalisation incestueuse ?» [13, p. 11].

\section{3. Épreuves projectives : entre actualisation des traumatismes sexuels et pubertaires}

À la croisée des problématiques du féminin à l'adolescence et de l'abus sexuel, nous retiendrons trois recherches utilisant la méthodologie projective : celle de Martin et al. [10], de Chagnon [5], et de Montreuil et al. [11]. Martin, Bloch-Lainé et al. ou encore Chagnon repèrent dans les Rorschach d'adolescentes non consultantes l'existence d'un espace psychique personnel, attestant une richesse de mentalisation favorisant la représentation de soi ; la présence de représentations au symbolisme sexuel marquée ; des défenses phobiques et rigides permettant de lier l'agressivité. Ces adolescentes dévoilent des représentations féminines-phalliques, ainsi que des réponses colorées de préoccupations pour l'enveloppe (parure, vêtement). Le surinvestissement de la féminité permet de tenir encore sous silence les désirs réceptifs et la rencontre avec la représentation sexuée de l'autre. Les modalités relationnelles évoquent des relations narcissiques, de type spéculaire (peu d'échanges

\footnotetext{
${ }^{1}$ Le rapport de la fille abusée à sa mère a été étudié notamment par Veyrat et Lefort [15].
} 
relationnels). Ainsi, les réponses « reflet » ou «symétrie » à la portée paradoxale ont-elles la fonction de maintenir la continuité narcissique par l'investissement d'un autre soi-même. De plus, Martin et Bloch-Lainé [8] soulignent chez des non-consultantes que «l'investissement des positions actives phalliques reste trop important pour que les modalités passives du narcissisme féminin soient intégrées afin de confirmer le choix identificatoire [...] ${ }^{2}[10$, p. 92]. Dans la recherche de Montreuil et al. [11] portant sur des femmes abusées sexuellement, les réponses projectives révèlent une fragilité identitaire et de l'enveloppe corporelle (fragilité des limites entre le dedans et le dehors, réponses «pénétration»), une baisse de l'estime de soi, une actualisation du traumatisme sexuel exprimée par des représentations angoissantes, liées à la confrontation à l'imago masculine. Quels sont les aménagements défensifs de notre préadolescente, son investissement de la féminité augurant de l'intégration du féminin et de ses capacités futures d'accueil et de contenance active ?

\section{Cas clinique}

Célia est placée dans un établissement social à la suite d'une mesure judiciaire. Des négligences éducatives (absentéisme scolaire et alcoolisme massif du père) entre 3 ans (date à laquelle sa maman décède de maladie) et 6 ans avaient motivé une mesure éducative en milieu ouvert, au cours de laquelle ont été révélés et dénoncés des abus sexuels répétés. Après un placement en famille d'accueil de quelques années, elle est prise en charge chez une de ses sœurs, dont la grossesse, mal vécue par Célia, provoque de nouveau une rupture familiale et motive un placement dans un foyer. La demande de rencontre et d'évaluation psychologiques est venue de l'équipe éducative qui note certains comportements interpellant : repli sur soi, difficultés relationnelles avec ses pairs qu'elle dénie, et à d'autres moments provocations, rabaissement de l'autre. Ainsi, apparaît-elle tour à tour rejetée et rejetante. Célia est l'objet de moqueries, et dans des moments de désespoir, a écrit sur son bras des insultes au cutter et au compas : la rage éprouvée à l'encontre des autres s'est retournée en conduite autoagressive. De cette autoagression, elle dira n'avoir rien senti.

En entretien, alternent des mouvements de compliance enfantine vis-à-vis du clinicien, d'euphorie, et des moments de tension lorsqu'on aborde ses relations avec les pairs. Elle ne fait jamais référence durant les entretiens aux événements traumatiques de son histoire, mais exprime une forte agressivité à l'endroit d'une sœur aînée, «coupable » d'avoir quitté le domicile familial et de ne pas avoir subi (sous-entendues des violences sexuelles du père). La 
clinique révèle une défense dilatoire contre le désir et l'excitation provoquée par l'autre, une fermeture à toute sollicitation pulsionnelle et à toute représentation de la sexualité génitale. L'angoisse d'intrusion reste forte et manifeste, d'une part, depuis peu par des conduites encoprésiques qui sont devenues régulières plus l'intérêt et la sollicitude des adultes augmentent, et, d'autre part, par la lutte de Célia contre la passivité du féminin, par la fermeture de son corps et la rétention volontaire de ses matières. La représentation fécalisée de soi altère l'appui sur des ressources internes, capables d'étayer la subjectivation et le féminin naissants à un temps d'adolescence dont l'enjeu est la représentation différenciée de soi, mais aussi la représentation d'un autre, à la fois pénétrant et comblant, menaçant et gratifiant. Fermer toute issue conduit à empêcher la perte du bon objet mais aussi la symbolisation de ce qui entre.

\section{Données projectives à l'épreuve du féminin et de la féminité ${ }^{2}$}

La représentation de soi n'est pas soutenue par des réponses humaines largement dépassées par les réponses «animales ». D'autre part, les contenus relevant d'un registre inanimé (12 réponses) soulignent une fixation à des éléments archaïques, embryonnaires et stagnants (racine, reflet), dans un protocole qui reste peu animé, non impliqué, comme si la projection était gelée. Les représentations sexuées sont précisément évitées. La planche VII, à résonance maternelle et féminine, induit une réponse dégradée, à connotation dysphorique, confirmée par une remarque subjective lors de l'enquête et de l'épreuve des choix (planche rejetée) : «C'est un papillon avec des bouts d'ailes. Il n'est pas heureux et déchiré. Elle n'est pas belle cette planche, elle est toute triste. »

$\mathrm{Au}$ regard des études cliniques précitées, ce protocole de Rorschach diffère de celui des populations d'adolescentes non consultantes. Nous signalons l'absence de réponse symbolique, des contenus peu variés, et le recours à des mécanismes défensifs inhibiteurs attestant un gel des capacités d'élaboration des représentations objectales. Nous repérons également l'absence d'attributs narcissiques phalliques connotant habituellement positivement l'image de soi. Aucune efflorescence de la féminité (fût-elle esquissée) dans ce protocole. À l'instar de l'étude menée par Condamin [6], le protocole de Rorschach produit par Célia ne témoigne pas de traces manifestes de l'agression et du trauma passés. Comme elle l'écrit ensuite en 2009 [7], la stratégie de l'oubli et du blanchiment s'avère payante, mais au prix 
d'un arasement de la personnalité et d'une enveloppe insuffisamment constituée et unifiée.

On retiendra du TAT de Célia et de ses scénographies la dramatisation des relations interpersonnelles en termes de destruction, de domination et de soumission. Les différents récits, se fixant d'abord au percept et à une réalité banalisée, s'achèvent le plus souvent dans des scènes de destruction, soulignant une grande hétérogénéité entre le début et la fin de l'histoire. Le protocole ici considéré met en évidence une mauvaise régulation de l'agressivité. Comme au Rorschach, le contrôle pulsionnel est renforcé par un accrochage au perceptuel et par l'investissement des limites, la dimension libidinale est neutralisée au profit de visions de violence subie, de menaces et d'abandon.

La mise en lien est soigneusement réprimée, évitée, ou bien s'abîme dans des explosions agressives, non contenues par des mouvements libidinaux. De plus, les planches convoquant la mise en tension des polarités féminine et maternelle mobilisent un fantasme victimaire. L'emprise, retrouvée aux planches mettant en scène la relation père/fille (6GF), mère/fille (7GF) et entre femmes (9GF), est une forme pervertie de lien, privant d'un support fiable et d'un contact authentique avec soi (supporter la régression) et avec l'autre.

\section{Gel, attente, ou attaque du féminin?}

Nous ne trouvons chez l'adolescente aucune déclaration critique ou d'acte de révolte contre le statut de femme et son indexation à une passivité réceptive, telle qu'elle peut être retrouvée chez des adolescentes comme rejeton de la revendication phallique. Les contenus à tonalité régressive renforcent une image de soi négative et le refus de l'engagement dans une relation complique le recours à des figures identificatoires structurantes. Les entretiens cliniques nous avaient révélé une préadolescente niant toute dépendance objectale mais ses déclarations d'autosuffisance. Le TAT reste marqué par la projection d'une violence non liée, mal intégrée dans des scénarios aux impasses destructrices, et surtout sans solution réparatrice. Dans les scénarios fantasmatiques, la haine s'échoue dans le meurtre, s'abîme dans l'inhibition après la projection des éléments hostiles du moi sur un objet reconnu comme féminin (si l'on se réfère aux planches du TAT), là où la trace du traumatisme sexuel ancien nous l'aurait fait placer du côté masculin. Sa problématique ne se situe pas au niveau du corps sexué, mais dans l'impossible intégration de la violence, laissant place à l'agressivité.

\footnotetext{
${ }^{2}$ Nous nous sommes référés aux travaux de l'École de Paris, et plus particulièrement à Chabert, Emmanuelli [4].
} 


\section{Conclusion}

La médiation du TAT et du Rorschach souligne la répression du féminin chez notre sujet et donne à voir une fragilité narcissique réactivée en présence de l'autre, ainsi qu'une fantasmatique mettant en scène un objet souffrant, sadisé par un objet jouissant. Les moyens de penser le traumatisme, accentué par l'adolescence, s'avèrent limités par l'accrochage au factuel - qui rétrécit la compétence interprétative - et par un clivage entre irruptions morbides et collage à la réalité. Comme l'exprime Ayoun [1], « Pour tenter de retrouver une "enveloppe psychique" en état de marche, certains sujets semblent s'agripper à la factualité [...]. Semblant fonctionner de manière hyperadaptée, mais isolés de tous. » Cette étude de cas conduit à une remarque méthodologique. En effet, les méthodes projectives, en tant qu'épreuves d'imagination induite, au-delà des protocoles issus de la procédure de passation standardisée, peuvent être prolongées par un travail d'association, voire par une mise en récit à partir tant des réponses au Rorschach que des histoires sollicitées par les planches du TAT. Par-delà la répétition et la remémoration, il s'agit au sens de Freud [8] d'initier un travail de perlaboration. En effet, prolonger le travail interprétatif à partir des supports projectifs nous met sur la voie de ce que J. Hochmann [9] nomme le «conte thérapeutique », cette coconstruction entre patient et praticien.

\section{Conflit d'intérêt : aucun}

\section{Références}

[1] Ayoun P. Vivre après le chaos incestueux (pas seulement survivre). Neuropsychiatrie de l'enfance et de l'adolescence 2008;56:269-72.

[2] Bernardi M, Peguet M. Les attaques de l'inceste contre l'activité de pensée. Travail psychologique du moment et vues rétrospectives. In: Castro D, editor. Incestes. Le Bouscat: L'esprit du temps; 1995. p.221-51.

[3] Bigras J, Balasc C. Ce qui se joue dans l'inceste père-fille : remarques sur la destruction d'une identité. Nervure 1988;1:54-64.

[4] Chabert C, Emmanuelli M. Interprétation des épreuves projectives à l'adolescence. EMC 2001; Psychiatrie/Pédopsychiatrie, 37-213-B-10.

[5] Chagnon JY. Féminité entre latence et adolescence. Adolescence 2005;23:557-73.

[6] Condamin C. Corps démembré, corps supplicié, corps massacré. Le Rorschach chez les 
enfants et adolescents victimes d'agression. Champ Psychosomatique 2006;41:129-42.

[7] Condamin C. Trauma, résilience et mémoire du corps. À propos d'un cas d'enfant sexuellement maltraité, approche projective et théorico-clinique. Bulletin de psychologie 2009; tome 62 (5)/503: 457-66.

[8] Freud S. Remémoration, répétition, et élaboration. In: La technique psychanalytique. Paris: PUF, collection Quadrige Grands textes; 2007.

[9] Hochmann J. Pour soigner l'enfant autiste. Des contes à rêver debout. Paris: Odile Jacob, collection Opus: 1997.

[10] Martin M, Bloch-Lainé F, et al. La féminité à l'adolescence : renoncement ou conquête. Psychologie clinique et projective 1995;1:87-96.

[11] Montreuil M, et al. Inceste et maltraitance : étude comparative au Rorschach. Psychologie clinique et projective 1996;1:31-47.

[12] Schaeffer J. Le refus du féminin. Paris: PUF (3 édition); 2007.

[13] Schaeffer J. Antagonisme et réconciliation entre féminin et maternel. Dialogue 2005;169:5-17.

[14] Upchurch DM, Kusunoki Y. Associations between forced sex, sexual and protective practices and sexually transmitted diseases among a national sample of adolescent girls. Womens Health Issues 2004;14:75-84.

[15] Veyrat JG, Lefort S. La famille malade de l'inceste. Ann Méd Psychol 1993;151:161-6.

\section{Discussion}

Dr Veyrat - Cette très intéressante communication m'interpelle d'autant plus que j'ai beaucoup travaillé sur les «familles malades de l'inceste ».

Qu'en est-il de la mère, dans cette étude ? A-t-elle pu passer des tests projectifs ? Et qui a dénoncé le père abuseur ? Elle-même, avec l'ambivalence inhérente à cet acte ?

Réponse du Rapporteur - Je remercie le Dr Veyrat de son intervention. Effectivement je me suis référée à votre étude de 1992 qui aborde la relation à la mère, que certains auteurs comme Montreuil ont qualifié de «traumatisme en creux». Dans la situation de Célia, la maman est décédée quand l'enfant avait 3 ans et ce décès a coïncidé avec des négligences éducatives paternelles (alcoolisme et déscolarisation) et des abus sexuels à l'endroit de sa 
seconde fille et sur la dernière, Célia. La révélation a été effectuée par la sœur à une famille d'accueil qui prenait en charge de façon séquentielle les deux enfants. Puis cette sœur adulte a demandé la garde de sa jeune sœur avant que Célia ne soit de nouveau confiée à une famille d'accueil. Le conflit entre elles deux s'est accentué au moment de la grossesse de celle-ci, réactivant une possible angoisse d'abandon et des difficultés d'intériorisation de l'objet primaire chez Célia. La petite fille a de nouveau mis à mal l'accompagnement éducatif suite à une proposition de la famille d'accueil de l'adopter. Elle a été ensuite confiée dans le foyer éducatif où nous exerçons. Il est à noter la discontinuité de prises en charge qui ne favorisent pas la construction psychique et la confiance en l'adulte.

Pr Allilaire - Ma question porte sur l'utilisation qu'il est possible de faire des contenus révélés par les outils projectifs et la valorisation des matériaux psychologiques riches qu'ils apportent au clinicien, à des fins thérapeutiques.

Comment valoriser ces données dans la prise en charge psychothérapique ?

Réponse du Rapporteur - Je vous remercie de votre question et effectivement vous interrogez l'utilisation des données cliniques issues d'un examen psychologique et surtout sa transmission à une équipe éducative qui n'est pas forcément sensibilisée à nos modèles théoriques. Comment rendre compréhensibles les conduites psychiques des adolescents révélées par les projectifs ? Même si nous ne l'avons pas abordé ici, le fonctionnement cognitif de Célia lui permet d'acquérir et de mobiliser des connaissances alors que dans le cadre scolaire, les résultats sont assez bas. Des préoccupations psychiques liées à la tentative de maîtrise de la destructivité et de l'agressivité peuvent rendre compte de ce manque de mobilisation et d'implication scolaires. De plus, les relations interpersonnelles de Célia telles qu'elles se traduisent au Rorschach et au TAT sont grevées d'emprise, voire de cruauté et rappellent les comportements de l'adolescente envers l'autre, cherchant à dénier sa dépendance tout en provoquant paradoxalement son rejet (dénigrement, encoprésie). Nous avons pu travailler auprès de l'équipe pluridisciplinaire la question du lien de l'adulte à cette adolescente et la nécessité de ne pas répondre en miroir à ces attitudes (contre-violence institutionnelle) afin de pacifier ses représentations d'objet. Ces données ont pu être discutées avec la pédopsychiatre qui la suit au sein d'un CMPP et qui a pu relever également à l'instar des projectifs l'hétérogénéité des conduites en entretien thérapeutique entre recours au factuel, attitude lisse et débordements agressifs, explosions violentes. 
Dr Barrois - On peut effectivement déplorer la réticence des étudiants et de certains enseignants à l'égard des tests projectifs.

Ici, vous nous présentez un ensemble de lectures psychanalytiques (d'inspiration freudienne, kleinienne, de D. Anzieu, de Winnicott).

On peut espérer que cette patiente pourra bénéficier d'une psychothérapie analytique intensive, en institution, puis hors institution.

Réponse du Rapporteur - Je ne peux que partager votre remarque sur le refus ou du moins la réserve de certains collègues, des enseignants et des étudiants qui préfèrent les données soit plus quantitatives (questionnaires notamment) ou encore se contentent des entretiens alors même que l'évaluation du fonctionnement psychique du sujet nécessite la confrontation des hypothèses issus d'outils différenciés et spécifiques. Célia bénéficie depuis près d'une année d'un suivi thérapeutique mis en place à la suite de l'examen psychologique et l'équipe comme moi-même avons insisté sur la nécessité de médiatiser, de travailler le lien aux deux sœurs. Cette relation sororale ne peut actuellement fonctionner à trois, la triangulation n'est pas effective au profit de l'exclusion d'une des trois sœurs tour à tour ; la sœur aînée est par exemple coupable de ne pas avoir subi les manœuvres sexuelles de son père et Célia est rejetée au regard de son manque d'investissement scolaire et de ses conduites. Au regard des comportements et de l'usure de l'équipe et du risque que celle-ci ne soit plus réceptive au soutien psychologique amené lors des réunions, il est nécessaire dans ce type de prise en charge d'adolescents en errance, présentant de troubles du comportement ou aux conduites hétérogènes, qu'une institution quelle qu'elle soit ne soit pas seule à assurer l'accompagnement éducatif et d'être soutenu dans l'élaboration du projet de vie par d'autres institutions partenaires de façon étayante et soutenue. 